\title{
Case report: Real-time contrast-enhanced ultrasonography for the diagnosis of typical 'bull's eye' sign of hepatic abscess caused by Acinetobacter baumannii in a tumor patient
}

\author{
MENG WU and QI-CHAO ZHENG \\ Department of Ultrasonography, Zhongnan Hospital of Wuhan University, Wuhan, Hubei 430071, P.R. China
}

Received January 22, 2015; Accepted September 25, 2015

DOI: $10.3892 /$ etm.2016.2976

\begin{abstract}
The present case report details a unique case of a 51-year-old male patient who underwent a radical operation for carcinoma of the stomach and pancreaticoduodenectomy. Conventional ultrasonography examination exhibited a characteristic 'bull's eye' sign in the liver; whereas real-time contrast-enhanced ultrasonography (CEUS) demonstrated the patient was suffering from a hepatic abscess, corroborated by cytologic examination, which confirmed the presence of Acinetobacter baumannii. The hepatic localization of A. baumannii is rare in tumor patients presenting with a typical 'bull's eye' sign; and such a case could easily be misdiagnosed as hepatic metastasis. The findings presented in this case report demonstrate that real-time CEUS may offer important diagnostic elements, albeit not specific, which should, together with a positive cytologic test, confirm the diagnosis of a hepatic abscess.
\end{abstract}

\section{Introduction}

Liver abscess is type of liver suppurative lesion caused by microorganisms such as bacteria, fungus or Entamoeba histolytica. The mortality rate may reach $10-30 \%$ if liver abscess patients fail to receive timely treatment (1). Generally, liver abscesses are catalogued into three types: Bacterial liver abscess, mixed infection by numerous kind of bacterial, comprise $\sim 80 \%$ of liver abscess cases; $E$. histolytica-induced abscesses account for $10 \%$ of cases; and fungus-induced liver abscesses account for $<10 \%$ of cases $(2,3)$. Approximately $8-16 / 100,000$ of the total number of people who were admitted to hospital are caused by liver abscess (4). The majority of liver abscess patient are 60-70 years old. Similar rates of liver abscesses are exhibited by males and females; however, female patients

Correspondence to: Associate Professor Qi-Chao Zheng, Department of Ultrasonography, Zhongnan Hospital of Wuhan University, 169 Donghu Road, Wuhan, Hubei 430071, P.R. China E-mail: 81446803@qq.com

Key words: hepatic abscess, 'bull's eye' sign, contrast-enhanced ultrasonography typically have an improved prognosis compared with males. The most common causes of fatality in patients with liver abscess include septicopyemia, multiple organ failure and liver failure. Treatment options for bacterial liver abscesses include antibiotics, alone or in combination with percutaneous catheter drainage, surgical drainage or surgical resection. Treatment options for E. histolytica and fungus liver abscesses may conservatively include anti-amoeba and anti-fungal medication, or treatment measures similar to those for bacterial liver abscess. Hepatic abscesses are relatively common in Asian countries (5). However, the report of a typical 'bull's eye' sign hepatic abscess caused by Acinetobacter baumannii in a tumor patient is rare and could easily be to misdiagnosed as hepatic metastasis. During real-time contrast-enhanced ultrasonography (CEUS), lesions disappear with an intense and prolonged enhancement after contrast media injection. Typical contrast agents are microbubbles containing gas which are injected intravenously into the circulation. Ultrasonic imaging using microbubble contrast agents enhances the ultrasound backscatter, reflection of the ultrasound waves, in order to produce a sonogram with increased contrast due to the increased echogenicity difference (6-8). Once diagnosed, patients with hepatic abscesses typically recover following conservative surgical treatment or anti-inflammatory drainage therapy, and the characteristic 'bull's eye' sign in the liver gradually disappears $(9,10)$.

\section{Case report}

A 51-year-old male patient presented at the Zhongnan Hospital of Wuhan University (Wuhan, China) in October 2013 for treatment due to flatulence in the right upper abdomen and two days of fever. The patient experienced pain in the right upper abdomen due to the pressure. The patient had previously undergone a radical surgical operation for carcinoma of the stomach and pancreaticoduodenectomy. Written informed consent was obtained from the patient. Upon physical examination, the patient complained of deep tenderness in the right upper abdomen; however, no obvious masses were detected. Laboratory examinations (Liaison Analyzer; DiaSorin Deutschland GmbH, Germany) demonstrated that the patient's hepatic and renal functions and other biochemical indicators were normal; however, alpha-fetoprotein (AFP) and carcinoembryonic antigen (CEA) levels were higher than normal. 


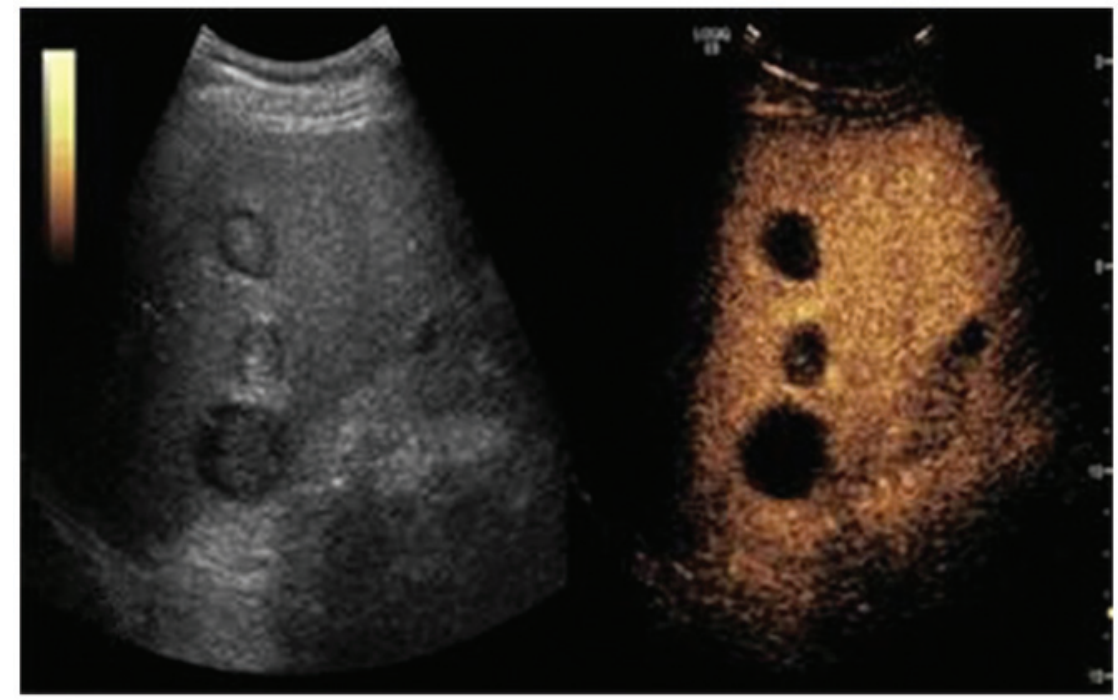

Figure 1. Examination with (left) conventional ultrasonography demonstrated a typical 'bulls-eye' sign; whereas (right) contrast-enhanced ultrasonography demonstrated that no filling enhancement was observed with the contrast agent in the whole focus.

A

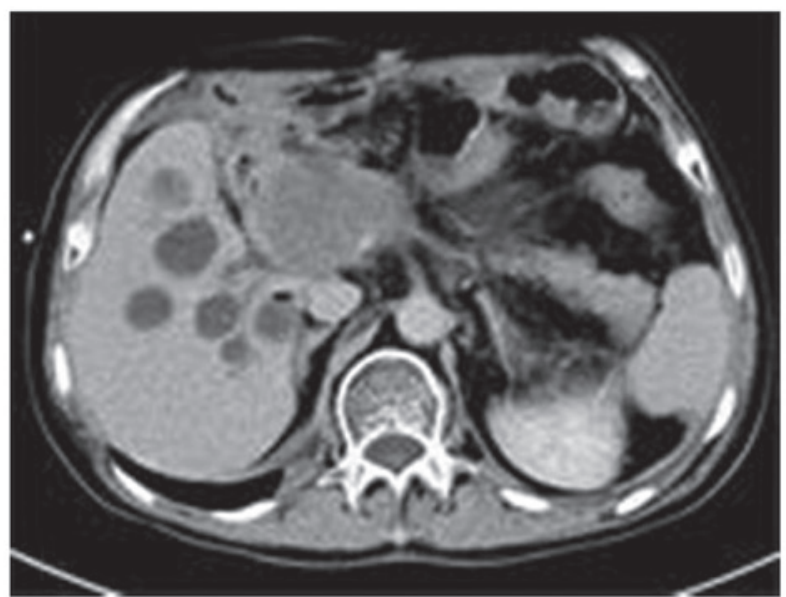

B

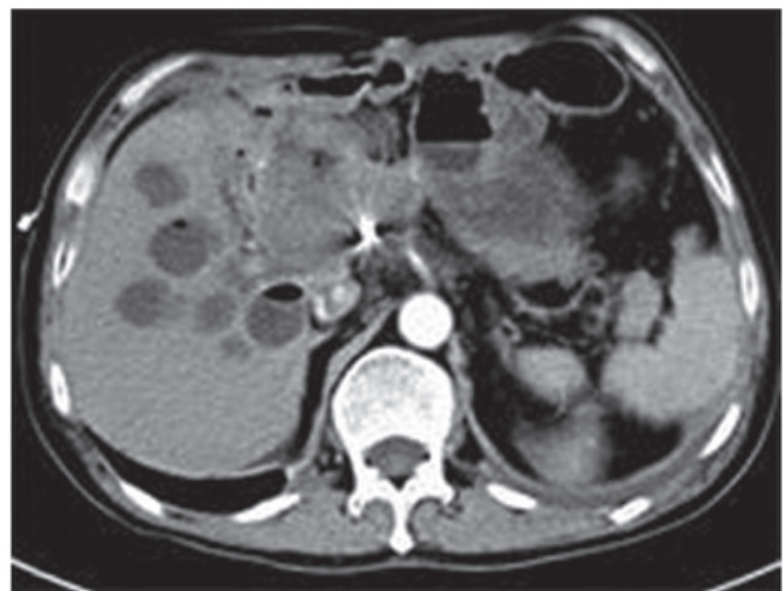

Figure 2. Diagnostic computed tomography (CT) scans. (A) Plain CT scan demonstrated several low echo nodules in the liver; whereas an (B) enhanced CT scan revealed no obvious enhancement of the hepatic nodules.

A

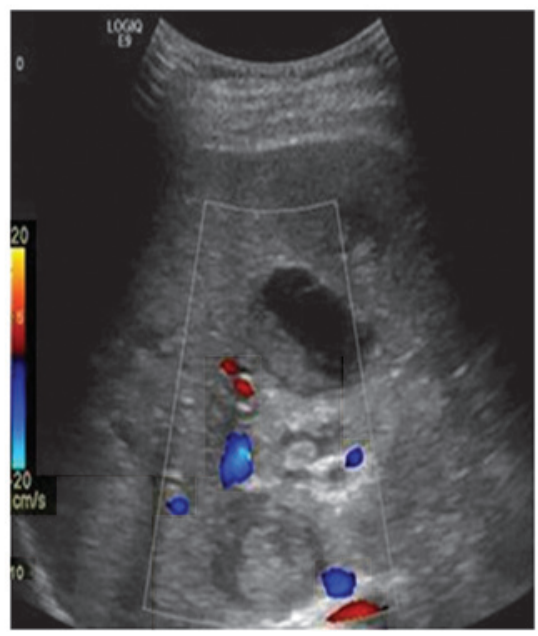

B

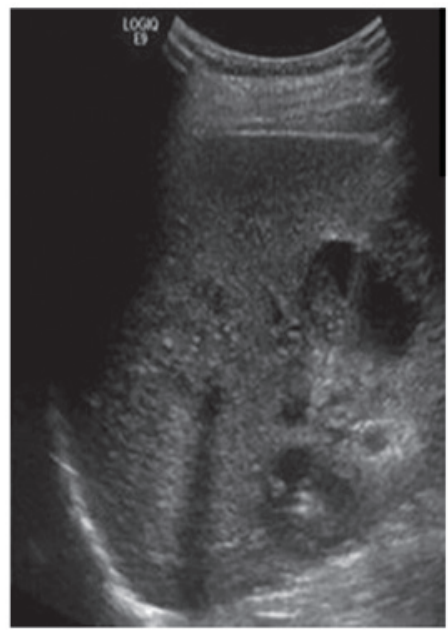

Figure 3. Patient liver after 5 days of anti-inflammatory therapy. (A) Color Doppler ultrasound scan, red represents blood flow directed to the probe, while blue represents blood flow away from the probe, indicating a small quantity of blood flow around the low echo nodule. (B) Conventional ultrasound scan demonstrating liquefaction in parts of the low echo nodule in the liver. Minimal blood flow could be seen around the low echo nodule. 


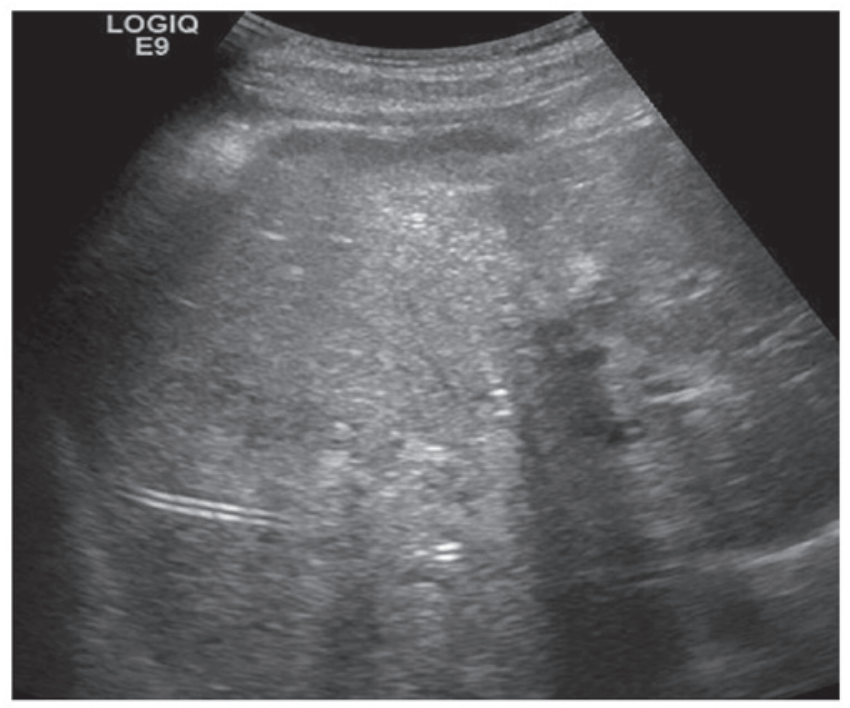

Figure 4. Following 30 days of drainage and anti-inflammatory therapy, a conventional ultrasonic scan demonstrated that the typical 'bulls-eye' sign in the liver had disappeared, the liver tissue had subsequently repaired and the patient gradually recovered.

Conventional and color Doppler ultrasonography were conducted using a GE E9 ultrasonography machine (GE Healthcare Life Sciences, Little Chalfont, UK) with an ultrasonography probe (probe, C1-C5; frequency, 2.0-5.0 MHz; mechanical index, 0.11). These examinations revealed several slightly low and slightly strong echoic masses in the liver with a surrounding low echo halo, representing a 'bull's eye' sign. The profile was clear, whereas the internal echoes were not; the size of one echo was $3.5 \times 2.5 \mathrm{~cm}$ and no obvious blood flow activity was demonstrated. These results did not indicate the presence of metastatic tumors.

Real-time CEUS was conducted using sulfur hexafluoride (SF6; SonoVue) as a contrast agent, with a mean microbubble diameter of $2.5 \mathrm{~mm}$ of the phospholipid microcyst. Real-time CEUS demonstrated that the arterial, portal vein and prolonged phases were not enhanced in the focal zone of the liver (Fig. 1). The ultrasound detected several cystic masses in the liver of the patient. These were considered to be benign lesions or hepatic abscesses, and a plain computed tomography (CT) scan (Spiral CT; Siemens, Munich, Germany) detected several low echo nodules in the liver (Fig. 2A). A number of nodules were detected; however, no obvious enhancement of the low echo nodule was observed following completion of an enhanced CT scan (Fig. 2B).

In order to make a definitive diagnosis, a sample was drawn from the liver by puncture with the guidance of traveling-wave ultrasonic sounds. A cytologic examination was completed on the grass green turbid liquid sample, which demonstrated that the sample contained Acinetobacter baumannii. Therefore, the final diagnosis was of hepatic abscess. Anti-inflammatory therapy was subsequently administered for 5 days, and liquefaction was detected in parts of the low echo nodule in the liver (Fig. 3). The therapy comprised of the intravenous administration of $3.0 \mathrm{~g}$ sulperzone and $0.9 \%$ normal saline every $8 \mathrm{~h}, 100 \mathrm{ml}$ metronidazole every $12 \mathrm{~h}$ and two tablets of tigecydine every $12 \mathrm{~h}$. Minimal blood flow was exhibited around the low echo nodule and, following 30 days of drainage and anti-inflammatory therapy, the characteristic 'bull's eye' sign in the liver disappeared. The hepatic tissue repaired and the patient gradually recovered (Fig. 4). AFP and CEA levels gradually returned to normal at a 5-month follow-up examination.

\section{Discussion}

Hepatic abscesses are common and are usually straightforward to clinically diagnose. In images generated through a conventional ultrasonic examination, a typical hepatic abscess presents as an even or uneven round low echo area with visible internal masses or spots $(11,12)$. However, following the increased consumption of clinical drugs, the manifestations of hepatic abscesses are increasingly atypical (13). Treatment for bacterial liver abscess may be conducted as follows: Prior to bacterial culture and drug sensitivity testing, intensified and large doses broad-spectrum antibiotic should be to eliminate gram-positive and gram-negative bacteria. In addition, anaerobion infection may occur, so anti-anaerobic agents should be applied regularly. Treatment for E. histolytica may be conducted as follows: Drugs to eliminate E. histolytica in the inner organs are commonly used, assisted by radical treatment drugs for the treatment of intestinal amebiasis $(3,14)$. Currently, metronidazole is the preferred antibiotic agent. A typical 'bull's eye' sign of hepatic abscess caused by A. baumannii may be observed in tumor patients, although this is considered an atypical hepatic abscess. Clinically, it is becoming increasingly difficult to decipher between atypical hepatic abscess and hepatic malignant tumors by conventional ultrasonic examination. As was demonstrated in the present case report, hepatic abscesses may exhibit a typical 'bull's eye' sign; however, in patients with a medical history of gastric carcinoma and colorectal cancer this may easily be misdiagnosed as metastatic colorectal cancer in the liver when using conventional ultrasonic examination. In the present case report, real-time CEUS was applied, as a supplement to the conventional ultrasonic examination, and this determined that the hepatic abscess exhibited no obvious enhancement in the arterial, portal vein or prolonged phases, which was inconsistent with the fast-in-fast-out imaging of hepatic malignant tumors. Furthermore, blood perfusion was observed on a real-time basis and the structure of the focal zone was clearly indicated. Based on these manifestations of real-time CEUS, an accurate ultrasonic diagnosis was achieved in this case. Therefore, the present case report demonstrated that real-time CEUS may provide great value for the definitive diagnosis of atypical hepatic abscesses, particularly in patients with a history of cancer, and also provides an important reference tool for differential diagnoses in clinical surgery.

\section{Acknowledgements}

This study was supported by the Youth Science Fund of Wuhan University (grant no. 2014A12).

\section{References}

1. Kuo SH,Lee YT, Li CR, Tseng CJ, Chao WN, Wang PH, Wong RH, Chen CC, Chen SC and Lee MC: Mortality in Emergency Department Sepsis score as prognostic indicator in patients with pyogenic liver abscess. Am J Emerg Med 31: 916-921, 2013. 
2. Sharma MP and Ahuja V: Amoebic Liver Abscess. JIACM 4: 107-111, 2003.

3. Sahoo AK and Rauta S: A clinical study on amoebic liver abscess. Bangladesh J Med Sci 14: 49-52, 2015.

4. Huang CJ, Pitt HA, Lipsett PA, Osterman FA, Lillemoe KD, Cameron JL and Zuidema GD: Pyogenic hepatic abscess. Changing trends over 42 years. Ann Surg 223: 600-609, 1996.

5. Rajagopalan S and Langer V: Hepatic abscesses. Med J Armed Forces India 68: 271-275, 2012.

6. Zhou K and Chen Z (eds): MRI of Body. Shanghai Medical University Press, Shanghai, pp888-892, 2000.

7. Claudon M, Cosgrove D, Albrecht T, Bolondi L, Bosio M Calliada F, Correas JM, Darge K Dietrich C, D'Onofrio M, et al: Guidelines and good clinical practice recommendations for contrast enhanced ultrasound (CEUS) - update 2008. Ultraschall Med 29: 28-44, 2008

8. Feinstein SB, Coll B, Staub D, Adam D, Schinkel AF, ten Cata FJ and Thomenius K: Contrast enhanced ultrasound imaging. J Nucl Cardio 17: 106-115, 2010.
9. Khanna S, Chaudhary D, Kumar A and Vij JC: Experience with aspiration in cases of amebic liver abscess in an endemic area. Eur J Clin Microbiol Infect Dis 24: 428-430, 2005.

10. Rajak CL, Gupta S, Jain S, Chawla Y, Gulati M and Suri S: Percutaneous treatment of liver abscesses: needle aspiration versus catheter drainage. AJR Am J Roentgenol 170: 1035-1039, 1998.

11. Donovan AJ, Yellin AE and Ralls PW: Hepatic abscess. World J Surg 15: 162-169, 1991.

12. Ryan RS, Al-Hashimi $\mathrm{H}$ and Lee MJ: Hepatic abscesses in elderly patients mimicking metastatic disease. Ir J Med Sci 170: 251-253, 2001.

13. Brown KT, Gandhi RT, Covey AM, Brody LA and Getrajdman GI: Pylephlebitis and liver abscess mimicking hepatocellular carcinoma. Hepatobiliary Pancreat Dis Int 2: 221-225, 2003.

14. Cerwenka H: Pyogenic liver abscess: Differences in etiology and treatment in Southeast Asia and Central Europe. World J Gastroenterol 16: 2458-2462, 2010. 